\title{
Domain-wall resonance in exchange-coupled magnetic films
}

\author{
R. L. Stamps* and A. S. Carriço \\ Departamento de Fisica, Centro de Ciencias Exatas, Universidade Federal do Rio Grande do Norte, 59072-970 Natal/RN, Brazil \\ P. E. Wigen \\ Department of Physics, Ohio State University, Columbus, Ohio 43210 \\ (Received 27 November 1995; revised manuscript received 9 July 1996)
}

\begin{abstract}
Exchange coupling between magnetic films in multilayer geometries can strongly influence magnetization behavior and spin-wave energies by correlating the motion of spins in one film with the motion of spins in adjacent films. In a similar fashion correlations can be expected between domain walls existing in neighboring exchange coupled films. In this paper we show that the motion of domain walls in neighboring films can depend strongly on interfilm exchange coupling. A static equilibrium configuration exists for the domain walls, and small amplitude oscillations about equilibrium can result in domain-wall resonances that involve interfilm exchange energies. Frequencies for optic- and acoustic-type domain-wall resonances are calculated and effects of a small static applied magnetic field are examined, revealing distinctly different behaviors for the acoustic and optic domain-wall resonances. The possibility for sensitive measurements of the local coupling by studying domain-wall resonance is discussed. Resonances with parallel and antiparallel coupling are considered and the response to a small in-plane driving field is calculated. [S0163-1829(97)00206-3]
\end{abstract}

\section{INTRODUCTION}

Numerous investigations of domain-wall motion and resonance have been made in various systems in order to study phenomena such as magnetization reversal, domain stability, and material homogeneity. In a similar vein, studies of domain configurations and domain walls in magnetic multilayers have provided valuable insights into the effects and character of interactions within and between magnetic layers. ${ }^{1}$ An interesting feature of multilayers are correlations between domain walls in separated films. ${ }^{2}$

To date, domain walls in multilayers have only been studied in terms of their static properties. Exchange coupling between domain walls on adjacent films can however lead to interesting dynamic effects. Consider two antiparallel coupled thin ferromagnetic films. The coupling energy will be smallest if walls in each film are positioned directly beneath one another as shown in Fig. 1(a) for two Néel walls. In this figure, the arrows represent the local orientation of the magnetization in each film. Small deviations from this configuration, depicted in Fig. 1(b), increase the energy of the structure and result in torques on the spins in the walls of each film. We will show in this paper how harmonic oscillations are possible with a natural frequency that depends on the magnitude and sign of the interfilm coupling.

Breathing oscillations of domain-wall pairs in single thin films have been predicted for certain wall configurations., In this paper, we consider a very different kind of restoring force responsible for wall oscillations-interlayer exchange coupling between magnetic films. In this regard it is useful to note that a continuing experimental problem is the quantitative measurement of interlayer magnetic coupling in layered magnetic film structures. ${ }^{5-7}$ The sign and average strength of the coupling are usually found through magnetization measurements, ferromagnetic resonance, light scattering, and magnetoresistance measurements. ${ }^{8-10}$ Coupled domain-wall resonance involving interlayer coupling as a restoring force thus offers interesting possibilities for new studies of interlayer exchange coupling.

In this paper we investigate the effect of coupling on dynamic correlations between domain walls in separate films and examine possibilities of using domain-wall resonance to study interlayer coupling on length scales the order of the width of a domain wall. In order to place the results for wall resonance in perspective, it is useful to review known results for spin-wave excitations in uniformly magnetized coupled

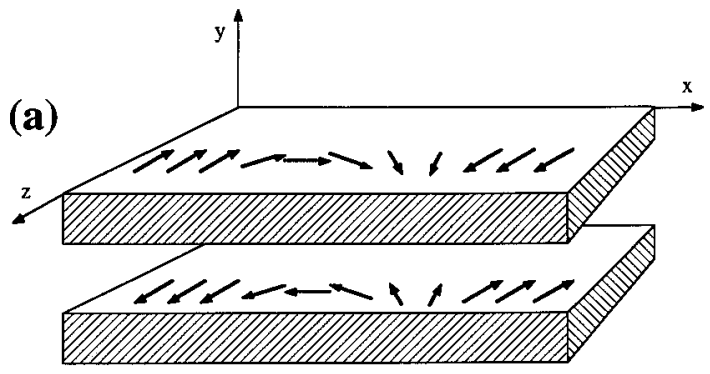

(b)

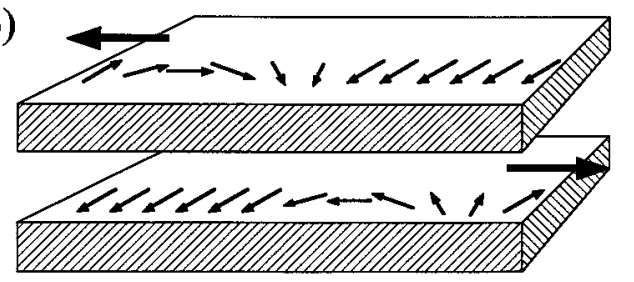

FIG. 1. Schematic illustration of relative motion and orientation of the magnetizations for Néel walls in two antiparallel coupled films. In (a) the equilibrium configuration is shown and in (b) walls are displaced a small amount with a corresponding increase in energy due to the coupling. The geometry is also defined with the $y$ axis normal to the film planes, the $x$ axis normal to the domain walls, and a static applied field in the $z$ direction. 
layers. For a sufficiently thin ferromagnetic film, only the lowest energy spin-wave branch is easily observed in Brillouin light scattering or ferromagnetic resonance measurements. The energy of this branch at zero wave vector vanishes in the limit of zero applied field unless anisotropies are present. A uniaxial in-plane anisotropy produces a gap typically on the order of a few hundred Oe for ferromagnets such as Fe or Co. Two such films coupled by an effective magnetic coupling will support combinations of spin-wave modes where the spins in each film precess either together in phase or out of phase. These modes are usually referred to as acoustic and optic. The difference in frequency between the optic and acoustic modes is determined by the effective exchange field coupling the films, in addition to any differences between effective internal fields acting in each film.

Similar behavior can be expected for domain-wall resonance in coupled multilayers. Domain-wall resonance differs from spin-wave resonance, however, in that the precession of spins in the domain walls results in a translation of the domain wall along the film. Wall resonance frequencies are therefore determined by inertia involved in the translation and the nature of restoring "forces" incurred by the relative motion of the walls. In multilayers, interfilm coupling can act as a restoring force on each domain wall. The motion of the walls can then be thought of as similar to two masses connected by a spring. Acoustic- and optic-type oscillations are possible with an effective mass for each wall determined by interfilm coupling and magnetostatic energies. The oscillation frequencies follow directly as a ratio of the restoring force to the effective mass. Because magnetic anisotropy determines the wall profile, and walls are able to move without changing shape, a result is that the frequencies for wall resonance do not contain the anisotropy gap of spin-wave resonance and are usually much smaller than spin-wave frequencies.

Since the domain-wall resonance is due to restoring forces localized to the region of the domain wall, the frequencies of the resonances are determined by coupling across areas with dimensions determined by the domain-wall widths. These lengths are on the order of $100 \AA$ in high anisotropy ferromagnetic metals. Observation of domain-wall resonances would therefore allow for investigations of coupling mechanisms on a much smaller length scale than possible with ferromagnetic resonance or Brillouin light scattering. These measurement techniques provide values for the interfilm coupling averaged over lengths determined by the wavelength of the probing microwave or optical field, which puts the length scales at $1000 \AA$ lengths and more.

A picture of domain-wall resonance is easily expressed by formulating the problem of domain-wall resonance in the coupled film structure in terms of exchange, dipolar, and magnetic anisotropy contributions to the domain-wall energy. We do this in the next section where coupled equations of motion for each wall are derived and solved for the allowed frequencies. In Sec. III, we examine the effects of a small static applied magnetic field on the walls and wall resonance frequencies. Finally in Sec. IV, we describe an alternate formulation of the problem in order to provide a unified description of spin-wave and wall resonance excitations and derive response functions to an ac driving field.

\section{DOMAIN-WALL RESONANCE FOR COUPLED FILMS}

The geometry is shown in Fig. 1 with the $y$ direction normal to the film planes. The films have identical magnetic properties and are assumed to be very thin so that Néel-type walls are preferred and the wall profile is assumed to depend only on $x$. At equilibrium the spins lie in the $x z$ plane and far from the walls the magnetization of each film is collinear with the $z$ axis. Angles $\theta$ and $\phi$ are used to specify the orientation of the magnetization as a function of $x$ in each film. For the magnetization $\mathbf{m}_{1}$ in film 1 , the components are

$$
\begin{gathered}
m_{1 x}(x)=\mathbf{M} \sin \left(\theta_{1}(x)\right) \cos \left(\phi_{1}(x)\right), \\
m_{1 y}(x)=\mathbf{M} \sin \left(\theta_{1}(x)\right) \sin \left(\phi_{1}(x)\right), \\
m_{1 z}(x)=\mathbf{M} \cos \left(\theta_{1}(x)\right)
\end{gathered}
$$

$\mathbf{M}$ is the magnitude of the magnetization vector and assumed to be the same in both films. A magnetization $\mathbf{m}_{2}(x)$ for the second film is defined similarly using $\theta_{2}(x)$ and $\phi_{2}(x)$.

The parameters entering into the wall energy are as follows. A uniaxial anisotropy $K$ with an easy axis along the $z$ direction is assumed for each film. The magnitude of the intrafilm exchange energy is specified with a constant $A$ and the interfilm coupling has strength $J$ (here $J$ is averaged over the film thickness and is in units energy per volume). A small magnetic field $h$ is also applied in the $z$ direction. The energy per wall area of the two-film system in the continuum limit is given by

$$
\begin{aligned}
& E=\int\left\{A\left[\left(\frac{d \theta_{1}}{d x}\right)^{2}+\left(\frac{d \theta_{2}}{d x}\right)^{2}\right]+K\left[\sin ^{2} \theta_{1}+\sin ^{2} \theta_{2}\right]\right\} d x \\
& +2 \pi M^{2} \int\left[\sin ^{2} \theta_{1} \sin ^{2} \phi_{1}+\sin ^{2} \theta_{2} \sin ^{2} \phi_{2}\right] d x \\
& -p J \int\left\{\begin{array}{l}
\sin \theta_{1} \cos \phi_{1} \sin \theta_{2} \cos \phi_{2} \\
+\sin \theta_{1} \sin \phi_{1} \sin \theta_{2} \sin \phi_{2}+\cos \theta_{1} \cos \theta_{2}
\end{array}\right\} d x \\
& -M h \int\left[\cos \theta_{1}+\cos \theta_{2}\right] d x \text {. }
\end{aligned}
$$

Our notation is chosen so that $J>0$ always. We use $p$ to denote the type of coupling by defining $p=+1$ to mean parallel coupling and $p=-1$ to mean antiparallel coupling.

The first set of terms contain the intrafilm exchange and uniaxial anisotropy that determine the shape and energy of the uncoupled domain walls in film. We note that magnetostatic energies due to the divergence of the magnetization in the film plane can be approximated by including a positiondependent demagnetizing energy that varies as $\sin ^{2} \theta$. This has the same functional form as the uniaxial anisotropy and is included in the definition of $K$. The second set of terms in Eq. (4) are demagnetizing energies for out-of-plane fluctuations. The third and fourth integrals are the interfilm coupling energy and the Zeeman energy in an applied field $h$.

The magnetostatic energies are assumed small in comparison to an uncoupled domain-wall energy per area $\sigma_{o}$ :

$$
\sigma_{o}=4[A K]^{1 / 2} .
$$


An approximate solution for the profiles specified by $\theta_{1}$, $\theta_{2}, \phi_{1}$, and $\phi_{2}$ are found by using a variational method involving trial solutions for the coupled equations. The trial solutions describe independent walls in uncoupled films. For parallel coupling these are given by

$$
\begin{aligned}
& \cos \theta_{1}=\tanh [(x) / \Delta] \quad \text { and } \phi_{1}=0, \\
& \cos \theta_{2}=\tanh [(x) / \Delta] \quad \text { and } \phi_{2}=0,
\end{aligned}
$$

and for antiparallel coupling, by

$$
\begin{gathered}
\cos \theta_{1}=\tanh [(x) / \Delta] \quad \text { and } \phi_{1}=0, \\
\cos \theta_{2}=-\tanh [(x) / \Delta] \quad \text { and } \phi_{2}=\pi .
\end{gathered}
$$

The wall width $\Delta$ is used as the variational parameter. The energy of the static coupled wall structure is then found by substituting the appropriate solutions from Eqs. (6)-(9), as determined by the sign of $p$, into Eq. (4) and minimizing with respect to the wall width $\Delta$. This procedure assumes that the wall profile in the coupled film system is described by functions of the form used in Eqs. (6)-(9). This is a good approximation as long as the interfilm coupling and magnetostatic interactions introduce only small corrections to the profile of the walls in the individual films. ${ }^{11}$ In the special case of no applied field, for example, the interfilm exchange coupling does not deform the wall profiles at all in the static configuration. In this case, the two walls are simply centered over one another. The profiles are then given by Eqs. (6)-(9) with $\Delta=\Delta_{o}$ where

$$
\Delta_{o}=[A / K]^{1 / 2} .
$$

Wall motion involves translations of $\theta_{1}$ and $\theta_{2}$ along the $x$ direction. Deviations of the walls from their equilibrium positions perturb the wall profiles and change the wall width so that it is no longer given by Eq. (10). If we know $\theta_{1}$ and $\theta_{2}$ at equilibrium, the effect of deviations $x_{1}$ and $x_{2}$ away from equilibrium can be written to first order in $x_{1}$ and $x_{2}$ as

$$
\begin{aligned}
& \theta_{1}=\theta_{1 o}+x_{1} \theta_{1 x}, \\
& \theta_{2}=\theta_{2 o}+x_{2} \theta_{2 x} .
\end{aligned}
$$

The profile at equilibrium is $\theta_{i o}$ and $\theta_{i x}=d \theta_{i} / d x$ is evaluated in the equilibrium configuration of the wall pair.

Fluctuations out of the film plane are given by deviations of $\phi_{1}$ and $\phi_{2}$ from their equilibrium values. With the fluctuations denoted by $\Psi_{1}$ and $\Psi_{2}$, then in the case of parallel coupling (where the equilibrium values of $\phi_{1}$ and $\phi_{2}$ are zero) we have

$$
\phi_{1}=\Psi_{1} \text { and } \phi_{2}=\Psi_{2} .
$$

For antiparallel coupling the equilibrium values of $\phi_{1}$ and $\phi_{2}$ are zero and $\pi$, so we use instead

$$
\phi_{1}=\Psi_{1} \text { and } \phi_{2}=\pi-\Psi_{2} .
$$

The approximations given by Eqs. (11)-(14) are substituted into the energy of Eq. (4) and the cos and sin terms expanded to second order in the variables $x_{1}, x_{2}, \Psi_{1}$, and $\Psi_{2}$. The resulting integrals over $x$ are then evaluated using the trial wall profile solutions and treated as functions of the variational parameter $\Delta$.

We consider first the case of zero applied field $h$. The integrals are straightforward and so only the results are presented. The energy per area $\sigma$ of the wall pair, quadratic in the fluctuation variables $x$ and $\Psi$, can be put in the form

$$
\begin{aligned}
\sigma= & 4[A / \Delta+K \Delta]+4 \pi M^{2} \Delta\left[\left(\Psi_{1}\right)^{2}+\left(\Psi_{2}\right)^{2}\right] \\
& +J \Delta\left\{2 \Psi_{1} \Psi_{2}-p\left[\left(\Psi_{1}\right)^{2}+\left(\Psi_{2}\right)^{2}\right]\right\}+J\left(x_{1}-x_{2}\right)^{2} / \Delta .
\end{aligned}
$$

This energy is measured with respect to the total interfilm coupling energy for uniformly magnetized films, $J_{\text {tot }}=\mathrm{J} \int \mathrm{dx}$.

The last term of Eq. (15) shows how wall separations involve interlayer exchange coupling. This means that an interlayer exchange restoring force can exist so that domainwall resonance is possible without considering any other additional restoring forces. This is in contrast to domain-wall resonance in single films which requires some sort of additional restoring force due, for example, to pinning by defects. Note however that an interlayer exchange coupling restoring force only makes sense when the walls overlap such that $\left|x_{1}-x_{2}\right|<\Delta$. For the remainder of the paper we consider only cases where the amplitude of the wall oscillation is small so that this condition is fulfilled.

In order to calculate the allowed resonance frequencies, it is useful to include terms in the energy that describe possible additional restoring forces. Pinning effects due, for example, to inhomogeneities in the films can result in effective restoring forces. We treat these effects in an approximate manner ${ }^{11,12}$ by including terms in the wall energy proportional to $x^{2}$. This approximation is only useful for displacements from a defect smaller than the wall width since the quadratic form is not an accurate representation of the pinning potential far from a point defect. This is acceptable because our entire discussion is valid only for small amplitude oscillations of the walls.

Magnetostatic energies also enter the problem as a contribution to the effective coupling between the two walls. ${ }^{13,14}$ The dipole interaction is long ranged and an accurate description of dipolar effects between coupled film domain walls is quite involved. Qualitatively, antiparallel alignment of the magnetization is preferred by stray magnetic fields between the films due the divergence of the magnetization within each film. This acts as an additional attractive force when the walls overlap. Parallel alignment, in contrast, is repulsive when the walls overlap. In order to represent these effects, we restrict our calculations to structures where magnetostatic energies are weak compared to the interlayer coupling and consider only cases where the walls overlap. Because the magnetostatic interaction between the walls will either add or subtract from the magnitude of the effective coupling correlating the wall motion, we represent the average magnetostatic contribution by an additional small quadratic dependence on wall separation $\left(x_{1}-x_{2}\right)^{2}$.

Based on the above considerations, approximate effects of magnetostatic interactions and pinning potentials are expressed by postulating an energy $E_{D}$ of the form 


$$
E_{D}=\left(K_{D} / \Delta\right)\left[\left(x_{1}\right)^{2}+\left(x_{2}\right)^{2}\right]-p\left(K_{D^{\prime}} / \Delta\right)\left(x_{1}-x_{2}\right)^{2},
$$

where $K_{D}$ controls the pinning potential (assumed for simplicity to be the same in each film) and $K_{D^{\prime}}$ represents the strength of the magnetostatic interaction between domainwall pair. The sign $p$ on $K_{D^{\prime}}$ describes the relative orientation of induced poles on the walls in the two films.
The energy of Eq. (15) together with Eq. (16) is next minimized with respect to the variational wall width. First, we set

$$
d\left(\sigma+E_{D}\right) / d \Delta=0
$$

in order to determine $\Delta$. The result is

$$
\Delta=\left\{4 A+\left(J-p K_{D^{\prime}}\right)\left(x_{1}-x_{2}\right)^{2}+K_{D}\left(x_{1}^{2}+x_{2}^{2}\right)\right\} /\left\{4 K+4 \pi M^{2}\left[\Psi_{1}^{2}+\Psi_{2}^{2}\right]+J\left(\Psi_{1}-p \Psi_{2}\right)^{2}\right\}^{1 / 2} .
$$

Equation (18) is then substituted into the wall energy per area $\sigma+E_{D}$ and terms to second order in the fluctuations $x$ and $\Psi$ are kept. The resulting expression for the energy is

$$
\begin{aligned}
\sigma= & 2 \sigma_{o}+4 \pi M^{2} \Delta_{o}\left[\left(\Psi_{1}\right)^{2}+\left(\Psi_{2}\right)^{2}\right]+J \Delta_{0}\left(\Psi_{1}-p \Psi_{2}\right)^{2} \\
& +\left(J-p K_{D^{\prime}}\right)\left(x_{1}-x_{2}\right)^{2} / \Delta_{o}+\left(K_{D} / \Delta_{0}\right)\left[\left(x_{1}\right)^{2}+\left(x_{2}\right)^{2}\right] .
\end{aligned}
$$

For the small displacements assumed here, the walls move rigidly so that the wall widths are simply given by $\Delta_{0}$. Also note that in the absence of fluctuations, the energy is simply that of two unperturbed walls.

The equations of motion can now be formed using the methods of Ref. 11 from the wall energy per area, Eq. (4), and appropriate wall profiles, Eqs. (6) and (7) or (8) and (9). We note that some care must be taken in determining the proper signs using the solutions in the different films. With $\sigma$ given in Eq. (19), the results are

$$
\begin{gathered}
(2 M / \gamma) d x_{i} / d t=d \sigma / d \Psi_{i}, \\
(2 M / \gamma) d \Psi_{i} / d t=-d \sigma / d x_{i} .
\end{gathered}
$$

$\gamma$ is the gyromagnetic ratio and the subscript " $i$ " identifies the film ( 1 or 2$)$. In order to solve these equations, time varying solutions of the form $\exp (-i \omega t)$ are assumed for $x_{1}$, $x_{2}, \Psi_{1}$, and $\Psi_{2}$. Substitution into Eqs. (20) and (21) results in a set of four linear coupled equations. Four allowed resonance frequencies $\omega$ can then be determined.

For parallel coupling, with $p=+1$, the frequencies are

$$
\left(\omega_{a} / \gamma\right)^{2}=4 \pi M H_{K}
$$

and

$$
\left(\omega_{o} / \gamma\right)^{2}=\left(H_{e}+4 \pi M\right)\left(H_{e}+H_{K}-H_{K^{\prime}}\right) .
$$

The effective fields appearing in Eqs. (22) and (23) are defined as follows:

$$
\begin{gathered}
H_{e}=2 J / M, \\
H_{K}=2 K_{D} / M, \\
H_{K^{\prime}}=2 K_{D^{\prime}} / M .
\end{gathered}
$$

The subscripts $a$ and $o$ refer to acoustic and optic and describe the relative phase between translations of the two walls. Note that the interfilm magnetostatic coupling repre- sented by $K_{D^{\prime}}$ reduces the optic mode frequency, as can be expected since in the parallel coupling configuration magnetostatic energies acting between the films are presumed to decrease the energy in Eq. (16).

The frequencies for parallel coupling are shown in Fig. 2 as functions of $H_{e}$ for different values of $H_{K^{\prime}}$. The results are given in unitless frequency and field variables defined by $\omega / \gamma 4 \pi M$ and $H_{e} / 4 \pi M$. In all cases $H_{K} / 4 \pi M=0.01$ which allows for a nonzero acoustic mode. Note that the acoustic mode is independent of $H_{e}$.

The main effect of magnetostatic interaction between the walls is to open a gap between the frequencies of the acoustic and optic resonances for $H_{e}=0$. Also note that competition between $H_{K^{\prime}}$ and the parallel $H_{e}$ coupling can be seen in Fig. 2. The magnetostatic repulsion lowers the frequency of the optic mode but leaves the acoustic mode unchanged. If $H_{e}+H_{K}-H_{K^{\prime}}<0$, magnetostatic energies overcome the attractive interlayer coupling and the domain walls repel each other. Thus we observe that the frequencies are nonzero and the structure is stable for $H_{K^{\prime}} / 4 \pi M$ less than 0.01 . The behavior of the domain-wall resonance in antiparallel coupled films is similar but differs in that the gap introduced by $H_{K^{\prime}}$ increases the frequency of the optic mode relative to the acoustic.

It is interesting to interpret the optic- and acoustic-mode

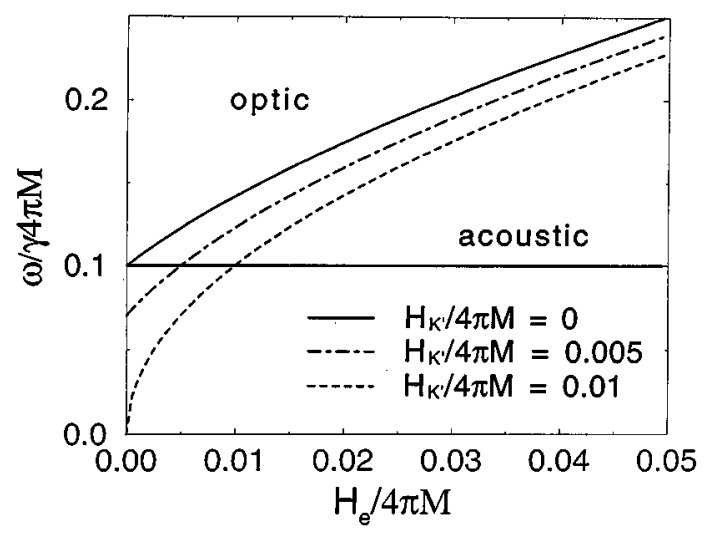

FIG. 2. Frequencies in zero applied field as a function of interfilm coupling strength. Frequencies for parallel coupling are shown for different values of $H_{K^{\prime}}$, which approximately represents a magnetostatic restoring force. $H_{K} / 4 \pi M=0.01$ in all cases. The frequency of the optic mode (solid line) is strongly dependent on the interlayer coupling. 
frequencies in terms of the harmonic oscillator analogy. The resonance frequency can be interpreted as a ratio between a restoring force and an effective mass. The effective mass in Eqs. (22) and (23) is the inverse of $4 \pi M$ for the acoustic mode and the inverse of $H_{e}+4 \pi M$ for the optic mode. The restoring forces are $H_{K}$ and $H_{K}-H_{K^{\prime}}+H_{e}$ for the acoustic and optic modes, respectively. The acoustic-mode frequency is identical to the resonance mode frequency of a single uncoupled wall given by $\left[4 \pi M H_{K}\right]^{1 / 2}$, with an effective mass that depends only on $M$ and a restoring force that depends only on restoring forces from pinning centers. The optic mode involves relative displacements of the walls with respect to one another, so that the restoring force includes contributions to the interlayer magnetic coupling $H_{e}$ in addition to effective forces due to $K_{D}$ and $K_{D^{\prime}}$. Note that when the films are uncoupled $\left(H_{K^{\prime}}=H_{e}=0\right)$ all frequencies reduce to the uncoupled single wall resonance frequency.

Two different modes also exist for antiparallel coupling with $p=-1$. These have frequencies:

$$
\left(\omega_{a} / \gamma\right)^{2}=\left(H_{e}+4 \pi M\right) H_{K}
$$

and

$$
\left(\omega_{o} / \gamma\right)^{2}=4 \pi M\left(H_{e}+H_{K}+H_{K^{\prime}}\right) .
$$

The acoustic-mode vanishes for both the parallel and antiparallel coupling if the only restoring force is interfilm coupling. When the interfilm coupling is zero and the films are uncoupled, both frequencies reduce to the single uncoupled wall resonance frequency. We also note that the effective masses are different for the two modes, as in the case of parallel coupling. In the present case, the acoustic-mode effective mass contains contributions from interlayer magnetic coupling whereas the optic-mode effective mass depends only on $M$.

\section{DOMAIN-WALL RESONANCE IN AN APPLIED STATIC FIELD}

We now consider domain-wall resonance for two antiparallel interlayer exchange coupled films in the presence of a small static applied field, $h=h_{s}$. For simplicity we neglect other energies represented by $K_{D}$ or $K_{D^{\prime}}$. The static applied field creates pressure on the walls and causes them to move apart. This is countered by antiparallel coupling that instead tries to bring the walls together. A static equilibrium exists with the walls positioned at $d_{1}$ and $d_{2}$ when the corresponding torques balance. The equilibrium positions $d_{1}$ and $d_{2}$ are found by minimizing the wall energy with respect to the position variables $x_{1}, x_{2}, \Psi_{1}$, and $\Psi_{2}$.

The frequencies of the wall resonance modes are unchanged when this is done using the energy per area of Eq. (4) expanded to second order in the $x$ and $\Psi$ variables. Field effects in the energies only appear for higher-order corrections to $\theta$ and $\phi$ in the energy. To see this, consider the expansion of the last term in Eq. (4) using Eqs. (11) and (12) with antiparallel coupling:

$$
\begin{aligned}
M h_{s} \int\left[\cos \theta_{1}+\cos \theta_{2}\right] d x \approx & -M h_{s} \int\left[\left(x_{1}-x_{2}\right) \theta_{x} \sin \theta_{o}\right. \\
& +(1 / 2)\left(x_{1}^{2}-x_{2}^{2}\right) \theta_{x}^{2} \cos \theta_{o}-(1 / 6) \\
& \left.\times\left(x_{1}^{3}-x_{2}^{3}\right) \theta_{x}^{3} \sin \theta_{o}\right] d x
\end{aligned}
$$

The $x^{2}$ terms vanish when the integrals in Eq. (29) are evaluated. The term linear in $x_{1}$ and $x_{2}$ determines the equilibrium position of the walls but does not enter the resonance frequencies. Only the $x^{3}$ terms enter into the frequencies by disturbing the wall profile and changing the wall width. Evaluation of integrals in Eq. (29) give the result

$$
\begin{aligned}
M h_{s} \int\left[\cos \theta_{1}+\cos \theta_{2}\right] d x \approx & -2 h_{s}\left[\left(x_{1}-x_{2}\right)\right. \\
& \left.-\left(x_{1}^{3}-x_{2}^{3}\right) /(3 \Delta)^{2}\right] .
\end{aligned}
$$

Only the third-order term involves the wall width $\Delta$. This, as will be shown below, leads to a field dependence of the wall width and consequently, the resonance frequencies.

The equilibrium separation between the walls is again found by setting $d \sigma / d x_{1}$ and $d \sigma / d x_{2}$ to zero. When the interfilm coupling is antiparallel, the equilibrium $x$ values are $x_{1}=-x_{2}=d$ with

$$
d / \Delta=\left(3 J / M h_{s}\right)\left\{1 \pm\left[1-(1 / 3)\left(M h_{s} / J\right)^{2}\right]^{1 / 2}\right\} .
$$

Our discussion assumes that $d$ is less than $\Delta$, and so we are only interested in the behavior for small $h$. The - root gives the proper behavior of $d$ for small $h_{s}$ whereas the + root gives unphysical answers for vanishing $h_{s}$. We also note that the equilibrium values for $\Psi_{1}$ and $\Psi_{2}$, determined by $d \sigma / d \Psi_{1,2}=0$, are still zero even to third order in the expansion.

The separation is nearly linear in $h_{s}$ for a wide range of fields $h_{s}$. For $J$ much larger than $M h_{s}$,

$$
d / \Delta \approx M h_{s} / 2 J .
$$

This relationship is exactly what one finds when solving for the equilibrium position of the walls keeping only terms to second order in the energy.

We next transform the position variables in $\sigma$ according to $x_{1} \rightarrow x_{1}+d$ and $x_{2} \rightarrow x_{2}-d$. Minimizing the result with respect to $\Delta$ as before, we arrive at an expression for the wall energy

$$
\begin{aligned}
\sigma= & 2 \sigma_{h}+\Delta_{h}\left\{\left(J+4 \pi M^{2}\right)\left[\left(\Psi_{1}\right)^{2}+\left(\Psi_{2}\right)^{2}\right]+2 J \Psi_{1} \Psi_{2}\right\} \\
& +\left(1 / \Delta_{h}\right)\left\{\left(J+M^{2} h_{s}^{2} / 3 J\right)\left[\left(x_{1}\right)^{2}+\left(x_{2}\right)^{2}\right]-2 J x_{1} x_{2}\right\},
\end{aligned}
$$

where

$$
\begin{gathered}
\sigma_{h}=2\left[\sigma_{o}^{2}-4(A / J)\left(M h_{s}\right)^{2}\right]^{1 / 2}, \\
\Delta_{h}=\Delta_{o} /\left[1-\left(M h_{s}\right)^{2} /(4 J K)\right]^{1 / 2} .
\end{gathered}
$$

The field dependence of the wall width and energy are clearly shown in Eqs. (34) and (35) and are due entirely to the third-order term appearing in Eq. (29). Competition between the applied field and the antiparallel interfilm coupling causes a distortion of the wall, leading to the field depen- 


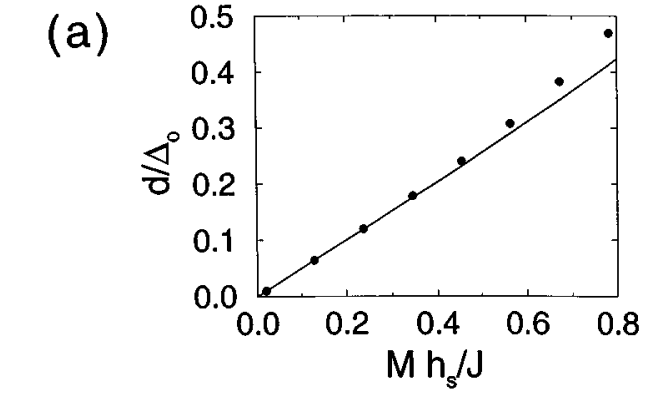

(b)

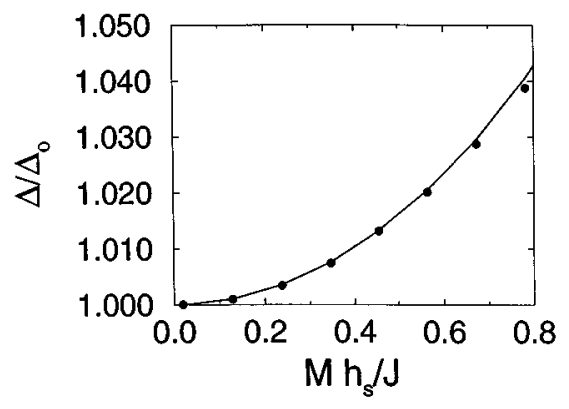

FIG. 3. Field dependence of (a) wall separation and (b) wall width. The solid lines are calculated using the analytical results of Eqs. (31) and (35) and the dots are from the numerical calculation described in Appendix A. Field dependence of the wall width enters through higher-order terms in the wall energy expansion.

dence of the wall width. As long as the walls overlap, the distortion of the walls increases as the separation increases.

It is interesting to examine the severity of the approximations made in deriving these equations. In Appendix A, a description is given of a numerical solution for the static configuration of two antiparallel coupled walls in an applied field. The results are shown in Fig. 3 along with the analytical approximation of Eqs. (31) and (35). The solid lines are the analytic results and the dots are the numerical results. In Fig. 3(a) the separation $d / \Delta_{o}$ is shown as a function of $M h_{s} / J$. The wall width $\Delta_{h} / \Delta_{o}$ is shown as a function of $M h_{s} / J$ in Fig. 3(b). In both cases, $J / K=0.5$ and $A / K=400$. The agreement is very good for small $h_{s}$, and only deteriorates for larger $h_{s}$.

The equations of motion are next constructed from the energy in Eq. (33) according to Eqs. (20) and (21). Solution of these give the following acoustic- and optic-mode frequencies:

$$
\left(\omega_{a} / \gamma\right)^{2}=\left(M h_{s}^{2} / 3 J\right)\left(H_{e}+4 \pi M\right)
$$

and

$$
\left(\omega_{o} / \gamma\right)^{2}=4 \pi M\left(H_{e}+M h_{s}^{2} / 3 J\right)
$$

We again emphasize that these results are valid in the antiparallel configuration and only for small wall separations less than a wall width. The striking feature is the existence of the acoustic mode for a small applied field. Reference to Eq. (27) shows that in the absence of an external applied field, the acoustic mode has zero frequency. This is because it takes no energy for the walls to translate equal amounts in the same direction so the acoustic-mode motion does not incur a restoring force. In the antiparallel configuration with a nonzero applied field, acoustic-mode motion always costs

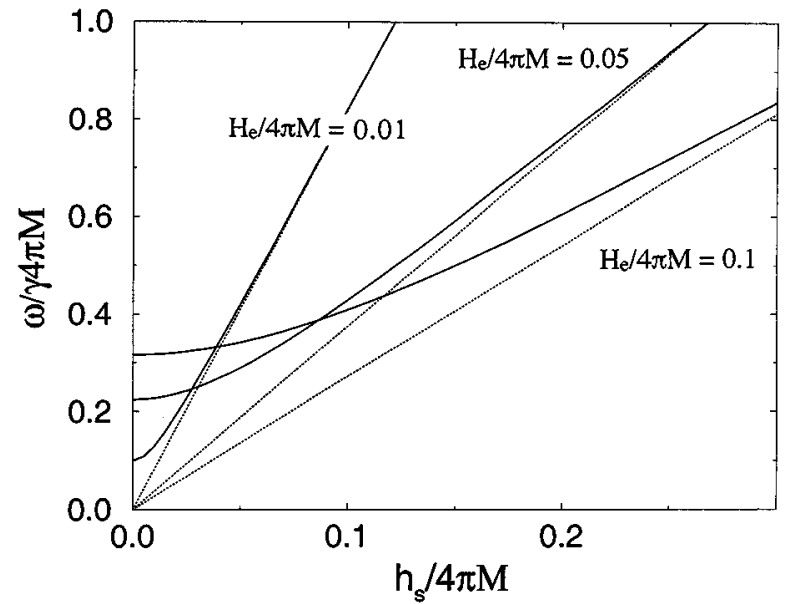

FIG. 4. Frequencies for antiparallel coupling as a function of applied field. The static applied field $h_{s}$ pushes the walls apart and the frequencies represent small oscillations about the equilibrium position. Note that the acoustic modes (dotted lines) increase linearly with field unlike the optic modes at small field strengths.

Zeeman energy for translation of one of the walls. Translation of the other wall in the acoustic mode gains Zeeman energy. This means that the two walls experience unequal forces with the result of a nonzero frequency for the acoustic mode, thus giving rise to the nonzero frequency of Eq. (36).

The optic-mode frequency is increased by the applied field, as can be seen by comparing Eqs. (28) and (37). The quadratic dependence on field is interesting because it means that field effects on the optic mode are only visible for large $h_{s}$. This is illustrated in Fig. 4 where wall resonance frequencies in the antiparallel configuration are shown as a function of field $h_{s}$. The dotted lines are the acoustic modes, $\omega_{a}$, and the solid lines are optic modes, $\omega_{o}$. The frequencies are given in dimensionless units as before with the field variable $h_{s} / 4 \pi M$. Coupling parameters $H_{e} / 4 \pi M$ between 0 and 0.1 are used. Note the linear dependence on $h_{s}$ for the acoustic-mode frequency whereas for small fields the opticmode frequency is insensitive to $h_{s}$. Also note that the sensitivity to changes in $h_{s}$ becomes less as the interlayer coupling $H_{e}$ is made stronger.

\section{RELATION TO SPIN WAVES AND DYNAMIC RESPONSE}

The theory presented in the previous sections was adequate for describing domain-wall profiles and motions in exchange-coupled films. In this section we construct a theory based on a discrete spin Hamiltonian capable of describing spin waves as well as domain-wall dynamics. This will allow a comparison of spin-wave frequencies and domain-wall resonances obtained using the same theory, and will also provide a general framework for constructing dynamic susceptibilities. Being a different approach, the theory of the present section can also be used as a check on the previous results.

We will see that a comparison of the frequencies for coupled film spin waves and domain-wall resonances provides a surprising result. As noted in the Introduction, anisotropies usually cause spin-wave frequencies to be larger 
than domain-wall resonance frequencies. In this section we show how interfilm exchange coupling can increase the optic domain-wall resonance frequency into the spin-wave region. The theory of this section will also be applied in the calculation of susceptibilities for small applied time varying magnetic fields.

Our approach is an extension of that used by Winter in describing wall waves in single Bloch walls. ${ }^{12}$ Because the formulation will begin with discrete variables, slightly different notations and geometry are used. To begin, the magnetic moments are not described by a continuous variable as before, but instead are assigned positions on a threedimensional periodic lattice with the index $i$. Spins in the top film 1 have amplitude $\mathbf{a}_{\mathbf{i}}$ and spins in the bottom film 2 have amplitude $\mathbf{b}_{\mathbf{i}}$.

The saturation magnetization in both films is $M$ and the anisotropy and exchange constants in each film are also assumed identical. The anisotropy $K$ is uniaxial as before with an easy axis in the $z$ direction. The geometry is the same as in Fig. 1 and the parameters and notation are as follows. Unlike the previous treatment, here a small time-dependent magnetic field $h$ is applied along the $z$ direction. The strength of the intrafilm exchange coupling between nearest neighbors is represented by the stiffness constant $D$. The two films are allowed to have different thicknesses; $t_{a}$ for the top film and $t_{b}$ for the bottom film.

Film thickness effects the strength of the interfilm exchange coupling experienced by the spins and can also control the strength of anisotropies in thin films. Out-of-plane anisotropies do not affect the profile of the in-plane walls assumed here, but they do influence the energies of spin wave and wall resonance excitations. The out-of-plane anisotropies enter the energy equations in the same manner as dipolar demagnetizing energies. We represent the total out-of-plane anisotropy with an effective perpendicular anisotropy, $K_{\text {eff }}$, defined for each film as

$$
K_{\text {eff }}^{a}=2 \pi M^{2}+K_{p} / t_{a}
$$

and

$$
K_{\text {eff }}^{b}=2 \pi M^{2}+K_{p} / t_{b},
$$

where $K_{p}$ is an out-of-plane uniaxial anisotropy with units energy per area. Anisotropies of this form often appear in thin films as a surface or interface anisotropy.

The energy per wall area for the system in this notation is

$$
\begin{aligned}
E= & -\frac{D}{M^{2}} \sum_{\langle i, j\rangle}\left[t_{a} \mathbf{a}_{i} \cdot \mathbf{a}_{j}+t_{b} \mathbf{b}_{i} \cdot \mathbf{b}_{j}\right]+\frac{K}{M^{2}} \sum_{i}\left\{t_{a}\left[\left(a_{i, x}\right)^{2}+\left(1+\frac{K_{\mathrm{eff}}^{a}}{K}\right)\left(a_{i, y}\right)^{2}\right]+t_{b}\left[\left(b_{i, x}\right)^{2}+\left(1+\frac{K_{\mathrm{eff}}^{b}}{K}\right)\left(b_{i, y}\right)^{2}\right]\right\} \\
& -h \sum_{i}\left[t_{a} a_{i, z}+t_{b} b_{1, z}\right]-p \frac{J}{M^{2}} \sum_{i} \mathbf{a}_{i} \cdot \mathbf{b}_{i} .
\end{aligned}
$$

The sums over $\langle i, j\rangle$ indicate sums over nearest neighbors. The first two sets of terms contain the exchange, anisotropy, and demagnetizing energies. Note that the uniaxial anisotropy $K$ is written here in terms of the $x$ and $y$ components of the magnetization rather than the $z$ component. This is more convenient for the present representation using variables in Cartesian coordinates. The third term represents the interaction energy with a driving field $h$. The last term represents the interfilm coupling which has the form of an effective Heisenberg interfilm exchange interaction. Note that the interfilm coupling has not been averaged over the film as before and has units energy per area. The parameter " $p$ " is used to specify parallel $(p=1)$ or antiparallel $(p=-1)$ coupling as before. Note also that the films are assumed to be very thin such that the magnetization is uniform across the film thickness. This is a reasonable approximation for strong ferromagnets such as $\mathrm{Fe}$ and $\mathrm{Co}$ for film thicknesses less than $50 \AA$.

The orientation of a magnetic moment at site $i$ is specified by an angle $\alpha_{i}$ in the top film and the angle $\beta_{i}$ in the bottom film. Both angles are referenced from the $z$ axis as shown in Fig. 5. The equilibrium positions of the magnetic moments are always assumed to be in the $x z$ plane because of demagnetizing fields in a thin-film geometry. The energy per area [Eq. (40)] is then written with the magnetic moments at each position $i$ rotated into a local coordinate system. The local $z^{\prime}$ axis defines the equilibrium orientation of the magnetic moment at site $i$. For example, $\mathbf{a}_{i}$ is rotated into $\mathbf{a}_{i}^{\prime}$ via

$$
\begin{gathered}
a_{i, x}^{\prime}=a_{i, x} \cos \alpha_{i}-a_{i, z} \sin \alpha_{i}, \\
a_{i, y}^{\prime}=a_{i, y}, \\
a_{i, z}^{\prime}=a_{i, x} \sin \alpha_{i}+a_{i, z} \sin \alpha_{i} .
\end{gathered}
$$

In our convention, primed variables are in the local system.

Before continuing, we also include a description of pinning effects as in previous sections. This is done in the present notation by including into the total energy an additional uniaxial anisotropy energy

$$
E_{u}=K_{D}\left(a_{x}^{\prime 2}+b_{x}^{\prime 2}\right) / M^{2} .
$$

This can be shown to result in a restoring force in the equations of motion of the same form used in Eq. (16). ${ }^{12}$ Magnetostatic effects, discussed in Sec. II, are ignored in the present calculation.

The next step in the calculation is to expand the $z$ components of the moments in terms of transverse fluctuations in the local $x$ and $y$ directions:

$$
a_{i, z}^{\prime} \approx M-\left[a_{i, x}^{\prime 2}+a_{i, y}^{\prime 2}\right] /(2 M)
$$




$$
b_{i, z}^{\prime} \approx M-\left[b_{i, x}^{\prime 2}+b_{i, y}^{\prime 2}\right] /(2 M) .
$$

The resulting energy describes second-order fluctuations in the spin variables that are discrete functions of position. Effective fields at a given site are next determined using $\mathbf{H}_{i}^{a}=-\nabla_{\mathbf{a}_{i}^{\prime}}\left(E+E_{u}\right)$ and $\mathbf{H}_{i}^{b}=-\nabla_{\mathbf{b}_{i}^{\prime}}\left(E+E_{u}\right)$. We are primarily concerned with long wavelength excitations and materials with wide domain walls so that $\mathbf{a}^{\prime}, \mathbf{b}^{\prime}, \alpha$, and $\beta$ vary little between neighboring lattice sites. We therefore transform the effective fields from a discrete form to a continuum limit with $\mathbf{a}_{i}^{\prime} \rightarrow \mathbf{a}^{\prime}(x)$ and $\mathbf{b}_{i}^{\prime} \rightarrow \mathbf{b}^{\prime}(x)$ in the usual manner. We note that in order to convert the discrete variables $\alpha_{i}$ and $\beta_{i}$ to continuous functions $\alpha(x)$ and $\beta(x)$ we expand quantities such as $\alpha(x+\delta)$, where $\delta$ is a nearest-neighbor distance, in a Taylor's expansion under the assumption that there is a domain-wall structure in the films. In particular, we use the relations

$$
\begin{aligned}
& d \alpha / d x=\sin \alpha / \Delta_{0}, \\
& d \beta / d x=\sin \beta / \Delta_{0} .
\end{aligned}
$$

In the following $D$ is contained in the exchange parameter $A$ which includes lattice constants and coordination numbers.

The final effective fields $\mathbf{H}^{a}$ and $\mathbf{H}^{b}$ are

$$
\begin{aligned}
H_{x}^{a}= & {\left[\frac{2 A}{M^{2}} \nabla^{2}-\frac{2 K}{M^{2}}\left(\cos ^{2} \alpha-\sin ^{2} \alpha\right)-\frac{2 K_{D}}{M^{2}}+p \frac{J_{a}}{M^{2}}\right] a_{x}^{\prime} } \\
& -\frac{J_{a}}{M^{2}} b_{x}^{\prime}+h \sin \alpha, \\
H_{y}^{a}= & {\left[\frac{2 A}{M^{2}} \nabla^{2}-\frac{2 K}{M^{2}}\left(\cos ^{2} \alpha-\sin ^{2} \alpha\right)-\frac{2 K_{\mathrm{eff}}^{a}}{M^{2}}+p \frac{J_{a}}{M^{2}}\right] a_{y}^{\prime} } \\
& -\frac{J_{a}}{M^{2}} b_{y}^{\prime}, \\
H_{x}^{b}= & {\left[\frac{2 A}{M^{2}} \nabla^{2}-\frac{2 K}{M^{2}}\left(\cos ^{2} \beta-\sin ^{2} \beta\right)-\frac{2 K_{D}}{M^{2}}+p \frac{J_{b}}{M^{2}}\right] b_{x}^{\prime} } \\
& -\frac{J_{b}}{M^{2}} a_{x}^{\prime}+h \sin \beta, \\
& -\frac{J_{b}}{M^{2}} a_{y}^{\prime}, \\
H_{y}^{a}= & {\left[\frac{2 A}{M^{2}} \nabla^{2}-\frac{2 K}{M^{2}}\left(\cos ^{2} \beta-\sin ^{2} \beta\right)-\frac{2 K_{\mathrm{eff}}^{b}}{M^{2}}+p \frac{J_{b}}{M^{2}}\right] b_{y}^{\prime} }
\end{aligned}
$$

The different film thicknesses scale the interfilm exchange appearing in each film and is represented by the quantities

$$
\begin{aligned}
& J_{a}=J / t_{a}, \\
& J_{b}=J / t_{b},
\end{aligned}
$$

in the expressions for the effective fields. We note that these expressions also agree with those of Winter in the case of zero interfilm coupling. ${ }^{12}$

Equations of motion are found with the usual torque equations

$$
\begin{aligned}
& \frac{d \mathbf{a}^{\prime}}{d t}=\gamma \mathbf{a}^{\prime} \times \mathbf{H}^{a}, \\
& \frac{d \mathbf{b}^{\prime}}{d t}=\gamma \mathbf{b}^{\prime} \times \mathbf{H}^{b} .
\end{aligned}
$$

The equations of motion given in Eq. (55) and (56) together with the effective fields given in Eqs. (49)-(52) are capable of describing both spin-wave and domain-wall dynamics in the coupled system. We consider zero wave-vector excitations for both spin-wave excitations and domain-wall translations. In each case we first calculate the frequencies for parallel and antiparallel coupling and then discuss the results in terms of response to the driving field $h$. The thicknesses $t_{a}$ and $t_{b}$ are set equal in the following discussion unless otherwise specified.

\section{Zero wave-vector spin waves}

In order to compare with results for spin waves in uniformly magnetized films, we set $K_{D}$ to zero. A time dependence for $\mathbf{a}^{\prime}, \mathbf{b}^{\prime}$, and $h$ of the form $\exp (-i \omega t)$ is assumed. Zero wave-vector spin-wave resonance solutions to the torque equations are

$$
\begin{aligned}
& a_{x}^{\prime}=A_{x} \tanh (x / \Delta) \exp (-i \omega t), \\
& a_{y}^{\prime}=A_{y} \tanh (x / \Delta) \exp (-i \omega t), \\
& b_{x}^{\prime}=B_{x} \tanh (x / \Delta) \exp (-i \omega t), \\
& b_{y}^{\prime}=B_{y} \tanh (x / \Delta) \exp (-i \omega t) .
\end{aligned}
$$

Substitution of these solutions into the equations of motion [Eqs. (55) and (56)] give a set of four coupled equations. These equations are shown in Appendix B, Eqs. (B1).

We set $p=+1$ for parallel coupling. The allowed frequencies are found by setting the determinate of the correspond-

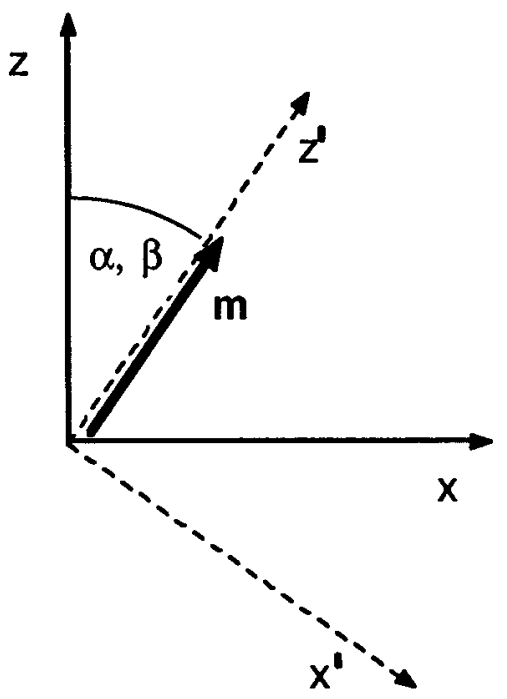

FIG. 5. In the discrete spin formulation, the coordinates are rotated locally in each film about the $y$ axis in order to place the primed $x^{\prime}$ and $z^{\prime}$ axes along the equilibrium directions of the local magnetizations. 
ing homogeneous (with $h=0$ ) set of equations to zero. Defining an effective exchange field $H_{e}$ as in Eq. (24), and effective anisotropy fields $H_{a}$ and $H_{\text {eff }}$ with

$$
\begin{gathered}
H_{a}=2 K / M, \\
H_{\mathrm{eff}}=2 K_{\mathrm{eff}} / M,
\end{gathered}
$$

the allowed frequencies are

$$
\omega_{o}^{2}=\gamma^{2}\left(H_{a}+H_{e}\right)\left(H_{a}+H_{e}+H_{\mathrm{eff}}\right)
$$

and

$$
\omega_{a}^{2}=\gamma^{2} H_{a}\left(H_{a}+H_{\mathrm{eff}}\right) .
$$

The subscripts $o$ and $a$ refer to "optic", and "acoustic", respectively, as before.

For antiparallel coupling, $p=-1$. The frequencies are

$$
\begin{gathered}
\omega_{o}^{2}=\gamma^{2}\left(H_{a}+H_{e}\right)\left(H_{a}+H_{\mathrm{eff}}\right), \\
\omega_{a}^{2}=\gamma^{2} H_{a}\left(H_{a}+H_{e}+H_{\mathrm{eff}}\right) .
\end{gathered}
$$

The results of Eqs. (63)-(66) agree with those of previous spin-wave and ferromagnetic resonance calculations on coupled films. ${ }^{8-10}$

The main points we now wish to emphasize are the difference in the optic-mode frequencies between parallel and antiparallel coupled cases and the existence of an energy "gap" which increases the frequencies of the excitations. The difference in frequencies between parallel and antiparallel is due to demagnetizing fields and the gap is due to the uniaxial in-plane anisotropy.

\section{Domain-wall resonance}

Solutions of the form

$$
\begin{aligned}
& a_{x}^{\prime}=A_{x} \sin \alpha \exp (-i \omega t), \\
& a_{y}^{\prime}=A_{y} \sin \alpha \exp (-i \omega t), \\
& b_{x}^{\prime}=B_{x} \sin \beta \exp (-i \omega t), \\
& b_{y}^{\prime}=B_{y} \sin \beta \exp (-i \omega t),
\end{aligned}
$$

correspond to translation of the domain wall in the $x$ direction. The corresponding equations of motion are shown in Appendix B in Eqs. (B2).

We allow $K_{D}$ to be nonzero and define a corresponding field $H_{K}$ as before. The frequencies found by setting the determinate of homogeneous $(h=0)$ set of equations to zero agree exactly with the $H_{K^{\prime}}=0$ form of Eqs. (22) and (23), for $p=+1$ and with Eqs. (27) and (28) for $p=-1$. Comparison of the spin-wave and domain-wall resonance frequencies show that the wall resonance frequencies are lower because of the absence of anisotropy terms in the frequencies.

Spin-wave resonance and wall resonance frequencies are shown in Figs. 6(a) and 6(b) as functions of exchange coupling. In both cases a small additional restoring force $K_{D}$ is included $\left(K_{D} / 2 \pi M^{2}=0.05\right)$ with frequencies for antiparallel coupling shown in (a) and frequencies for parallel coupling shown in (b). The other parameters are: $t_{a}=t_{b}$, $K / 2 \pi M^{2}=1.5$. (a)

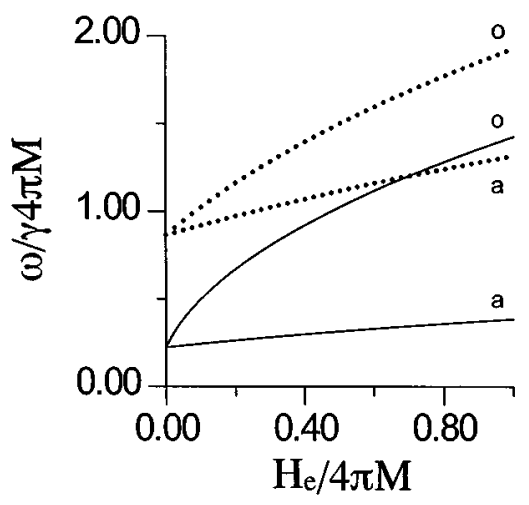

(b)

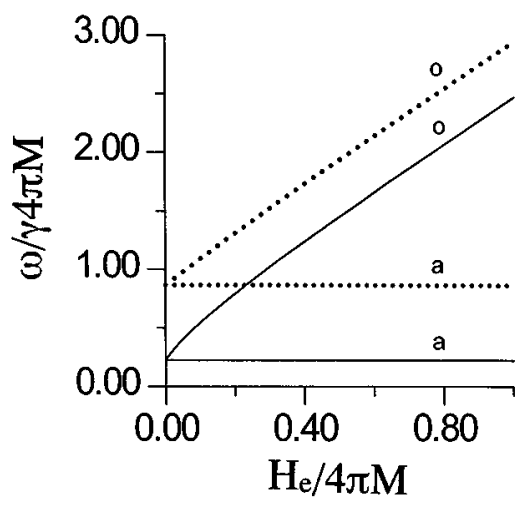

FIG. 6. Frequencies of the spin-wave and domain-wall resonances for the (a) antiparallel coupled and (b) parallel coupled configurations as functions of coupling strength $H_{e}$. The solid lines are acoustic (a) and optic (o) domain-wall resonances and the dotted lines are the acoustic and optic spin-wave resonances. The films are identical with anisotropies $K / 2 \pi M^{2}=1.5$ and $K_{p}=0$. A restoring force for the wall resonances is included with $K_{D} / 2 \pi M^{2}=0.05$. Note that the optic resonance mode has a frequency greater than the acoustic spin-wave mode for large $H_{e}$.

The difference in spin-wave resonance and wall resonance frequencies for both the parallel and antiparallel cases is due mostly to the in-plane uniaxial anisotropy $K$ with a small contribution from $K_{D}$. The acoustic modes are less sensitive to the interfilm exchange than the optic modes for antiparallel coupling and independent of the exchange for parallel coupling. Interestingly, a large interfilm exchange can cause the optic wall resonance mode to have a frequency greater than the acoustic spin-wave branch. This occurs for a smaller value of the exchange in the parallel case than in the antiparallel case.

As a final comment, we note that it is a simple matter to generalize the equations of motion in Eq. (B2) to describe domain-wall resonance in multilayers consisting of several coupled films. A band of wall resonances would appear, analogous to collective excitations in multilayers.

\section{Dynamic response}

As in ferromagnetic resonance on coupled films, standard techniques for observing domain-wall resonances in multi- 
layers require a net fluctuating magnetic moment for the coupled film structure. This is the situation for the optic wall resonance mode in antiparallel coupled films since out-ofphase translations lead to a change in the magnetization in the $z$ direction averaged over both films. This is not the case however for parallel coupled films since optic wall resonance translations lead to zero average change in the total magnetization.

This difficulty can be overcome by allowing $K_{\text {eff }}$ to be different for each film. This can be accomplished using different film thicknesses, i.e., $t_{a} \neq t_{b}$, as done by Zhang et al. ${ }^{7}$ in measurements of the acoustic and optic spin-wave resonances in multilayers with thickness dependent anisotropies. In order to gain a feeling for how the intensities of the wall resonance modes depend on unequal anisotropies in the presence of small rf driving fields, we calculate the response of the coupled wall structure to the time-dependent driving field $h$. The results are presented as averaged susceptibilities $\chi_{z z}$ and $\chi_{y z}$ that measure fluctuations in the $z$ and $y$ directions in the film geometry due to a driving field in the $z$ direction.

The susceptibilities are defined as averages over a film of length $L$ :

$$
\begin{aligned}
& \chi_{z z}=\frac{1}{h L} \int\left(A_{z}+B_{z}\right) d x, \\
& \chi_{y z}=\frac{1}{h L} \int\left(A_{y}+B_{y}\right) d x .
\end{aligned}
$$

The susceptibilities are found by solving the equations of motion [Eq. (B2)] for the amplitudes A and $\mathbf{B}$. For simplicity we present results with $K_{D}=0$. The general expressions are not particularly illuminating so it is useful instead to examine the susceptibilities for equal film thicknesses but with different $K_{\text {eff }}$. For antiparallel coupling the susceptibilities are

$$
\begin{gathered}
\chi_{z z}=\frac{\pi \Delta}{L} \frac{M\left(H_{\mathrm{eff}}^{a}+H_{\mathrm{eff}}^{b}\right)}{H_{e}\left(H_{\mathrm{eff}}^{a}+H_{\mathrm{eff}}^{b}\right) / 2-(\omega / \gamma)^{2}}, \\
\chi_{y z}=0 .
\end{gathered}
$$

In this case the $z$ susceptibility still has a pole at the wall resonances when $K_{\text {eff }}^{a}=K_{\text {eff }}^{b}$. Note that the total response defined by $\mathbf{a}+\mathbf{b}$ is only in the $z$ direction.

For parallel coupling we find

$$
\begin{gathered}
\chi_{z z}=\frac{\pi \Delta}{L} \frac{-M\left(H_{\mathrm{eff}}^{a}+H_{\mathrm{eff}}^{b}\right)}{(\omega / \gamma)^{2}\left[H_{e}^{2}+H_{e}\left(H_{\mathrm{eff}}^{a}+H_{\mathrm{eff}}^{b}\right) / 2-(\omega / \gamma)^{2}\right]} \\
\times\left[H_{e}^{2}+\frac{2 H_{e} H_{\mathrm{eff}}^{a} H_{\mathrm{eff}}^{b}}{H_{\mathrm{eff}}^{a}+H_{\mathrm{eff}}^{b}}-(\omega / \gamma)^{2}\right], \\
\chi_{y z}=\frac{2 i M}{\omega / \gamma} .
\end{gathered}
$$

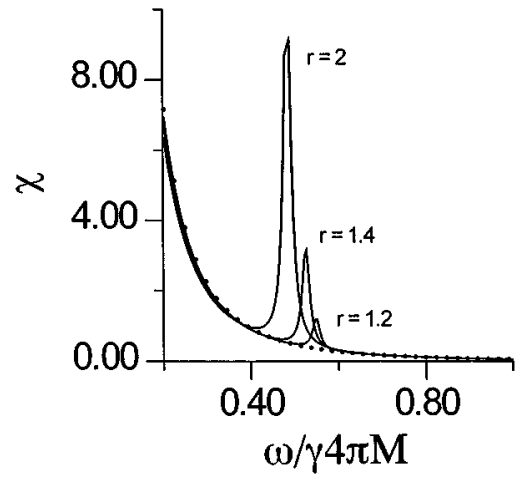

FIG. 7. Imaginary part $\chi_{x}^{\prime \prime}$ of the in-plane response $\chi_{x}$ as a function of driving field frequency for the parallel coupling configuration. The ratio $r$ controls the magnitude of the internal fields acting in film $b$ relative to those in film $a$ and the response is shown for four different $r$. The dotted line is the response for $r=1$. The parameters are $J / 2 \pi M^{2}=0.1, K / 2 \pi M^{2}=1.5, K_{p} / 2 \pi M^{2}=0.5$, and $K_{D} / 2 \pi M^{2}=0$. The response becomes larger with increasing $r$ while the peak response shifts lower in frequency as explained in the text.

Here again the resonance is only visible for the in-plane fluctuations of the magnetization. When $K_{\text {eff }}^{a}=K_{\text {eff }}^{b}$, then

$$
\chi_{z z}=\frac{-2 \pi \Delta M H_{\mathrm{eff}}}{L(\omega / \gamma)^{2}}
$$

and there is no longer a pole at the wall resonance in either component. This can be understood by considering the amplitude of precession for the magnetic moments in each film. When the effective fields acting within each film are the same, the precession amplitudes are the same. The walls then move equal amounts in the same direction, which takes no energy if $K_{D}=0$. There is then also no fluctuation in the net magnetization. When the effective internal fields are different for the two films, the two precession amplitudes are different and fluctuations in the net magnetization are possible.

The behavior of $\chi_{z z}$ for parallel coupling is illustrated in Fig. 7 for $t_{a} \neq t_{b}$. Here the susceptibility is found by solving the equations of motion for the sum of the in-plane fluctuations $A_{z}+B_{z}$. A small imaginary part was added to the frequency to give a width to the peaks in simulation of damping. We note that this should not be understood as properly representing actual damping processes.

The parameters for Fig. 7 are $K_{p} / 2 \pi M^{2}=0.5$, $J_{a} / 2 \pi M^{2}=0.1$, and $t_{a}$ held constant. The imaginary part $\chi_{z z}^{\prime \prime}$ of the response $\chi_{z z}$ is shown for three values of $r=t_{b} / t_{a}$ indicated on the plot with the dotted line calculated for $r=1$. As expected, the response to the optic wall resonance pole becomes larger as $r$ is increased. However the frequency at which the peak occurs decreases with increasing $r$. This decrease is due to the reduction of the internal fields as $t_{b}$ becomes larger, which weakens the interfilm coupling and anisotropies. Note also the large values of $\chi_{z z}^{\prime \prime}$ for small driving field frequencies due to the second-order pole at $\omega=0$.

\section{CONCLUSIONS}

We have examined spin-wave and domain-wall resonance in two thin ferromagnetic films coupled either parallel or 
antiparallel with an effective Heisenberg exchange interaction. The coupling acts as a restoring force correlating the wall motion in the separate films and only one wall resonance appears in the absence of other restoring forces. This resonance corresponds to an optic-type oscillation involving opposing motion of the walls. An acoustic-type oscillation appears when other restoring forces within the individual films exist.

For small interfilm coupling, the frequencies of the wall resonances lie below the frequencies for spin-wave resonance in the domain walls by an amount dependent on the in-plane anisotropy of the films. A feeling for the frequencies can be obtained by estimating the resonances for coupled ferromagnetic films such as Fe. With $4 \pi M=21 \mathrm{kG}, K=10^{6}$ $\mathrm{erg} / \mathrm{cm}^{3}, K_{p}=0$, and $H_{\mathrm{ex}}=0.1 \mathrm{kG}$ and in the absence of other restoring forces, the spin-wave resonance fields are 5.2 and $5.4 \mathrm{kG}$ for the acoustic and optic modes, respectively, in the antiparallel configuration. The optic wall resonance mode is at $1.45 \mathrm{kG}$. Domain-wall resonances in coupled transitionmetal magnetic structures should then occur in a range below $5 \mathrm{GHz}$ for weak to moderate coupling.

We also find that for larger interfilm coupling it is possible for the optic wall resonance to have a frequency comparable or even larger than the acoustic spin-wave resonance. This is interesting because it offers the possibility for large interactions between spin-wave and domain-wall excitations in strongly coupled films.

The effects of a small static applied magnetic field were examined for antiparallel coupling. The walls on the separate films separate until the pressure on the walls due to the field balances the pressure due to the antiparallel coupling. Both the acoustic- and the optic-mode oscillations about the equilibrium position of the walls are then possible. The acoustic mode increases linearly with field unlike the optic mode at small field strengths.

The possibility of observing domain-wall resonances in coupled film structures may allow the study of coupling mechanisms across regions with dimensions on the order of a domain-wall width. With experiments of this type in mind, we have examined the response of the domain-wall resonances to a small driving field. The optic wall resonance should be visible by looking at in-plane fluctuations of the magnetization if the films are antiparallel coupled. In the case of parallel coupling, the optic-mode resonance will only be visible if the effective internal fields acting in one film differ from those acting in the other film. Finally, we note that the theory presented in this paper applies to any layered magnetic system capable of supporting hard domain walls as long as the interlayer coupling is weak compared to intralayer coupling.

\section{ACKNOWLEDGMENTS}

We acknowledge stimulating discussions with A. Dantas and T. Dumelow. Work by R.L.S. was supported in part by the CNPq. R.L.S. also thanks the UFRN for support.

\section{APPENDIX A: NUMERICAL SOLUTION FOR STATIC CONFIGURATION}

Consider the case of antiparallel coupling $(p=-1)$. The energy per area given in Eq. (4) can be minimized by solving the corresponding Euler equations:

$$
2 A \frac{\partial^{2} \theta_{1}}{\partial x^{2}}-K \sin \left(2 \theta_{1}\right)+M h_{s} \sin \left(\theta_{1}\right)+J \sin \left(\theta_{1}-\theta_{2}\right)=0
$$

$$
2 A \frac{\partial^{2} \theta_{2}}{\partial x^{2}}-K \sin \left(2 \theta_{2}\right)+M h_{s} \sin \left(\theta_{2}\right)-J \sin \left(\theta_{1}-\theta_{2}\right)=0 .
$$

These can be written as two coupled finite difference equations where the value of $\theta$ at position $x$ is determined by values at neighboring positions a distance $\Delta x$ away at $x+\Delta x$ and $x-\Delta x$. These equations are

$$
\begin{aligned}
\theta_{1}(x)= & \frac{1}{2}\left[\theta_{1}(x+\Delta x)+\theta_{1}(x-\Delta x)\right]-\left(\frac{\Delta x}{2 \Delta_{0}}\right)^{2}\left[\sin \left[2 \theta_{1}(x)\right]\right. \\
& \left.-\frac{M h_{s}}{K} \sin \left[\theta_{1}(x)\right]-\frac{J}{K} \sin \left[\theta_{1}(x)-\theta_{2}(x)\right]\right], \quad \text { (A3) } \\
\theta_{2}(x)= & \frac{1}{2}\left[\theta_{2}(x+\Delta x)+\theta_{2}(x-\Delta x)\right]-\left(\frac{\Delta x}{2 \Delta_{0}}\right)^{2}\left[\sin \left[2 \theta_{2}(x)\right]\right. \\
& \left.-\frac{M h_{s}}{K} \sin \left[\theta_{2}(x)\right]+\frac{J}{K} \sin \left[\theta_{1}(x)-\theta_{2}(x)\right]\right] . \quad \text { (A4) }
\end{aligned}
$$

Numerical solution of these two equations is done by dividing a long interval in position $x$ into $N$ segments each of length $\Delta x$. Fixed values for $\theta_{1}$ and $\theta_{2}$ at the ends determine the boundary conditions. In this problem, the boundary conditions are $\theta_{1}=0, \theta_{2}=\pi$ in the first segment, and $\theta_{1}=\pi$, $\theta_{2}=2 \pi$ in the last segment. The values of $\theta_{1}$ and $\theta_{2}$ at each other segment in the interval are then adjusted according to Eqs. (A3) and (A4) iteratively. The values in each segment relax toward a solution of Eqs. (A1) and (A2) relatively quickly.

The solutions shown in Fig. 3 where obtained with 1000 iterations with $N=800$ and $\Delta x / \Delta_{0}=1 / 20$. The angles in this example converged within $10^{-4}$ radians and $N$ was chosen so that finite-size effects were negligible. The wall separation and width were determined by calculating the position and slope of the wall profiles where $\theta_{1}=\pi / 2$ and $\theta_{2}=3 \pi / 2$.

\section{APPENDIX B: EQUATIONS OF MOTION}

The torque equations Eqs. (55) and (56), using the effective fields given in Eqs. (49)-(52), are shown below. When solutions of the form presented in Eqs. (57)-(60) are used, equations of motion for spin-wave excitations on coupled domain walls result: 


$$
\left[\begin{array}{cccc}
i \omega / \gamma & 2 & 0 & \frac{J_{a}}{M} \\
-\frac{2}{M}\left[K+p J_{a}\right] & i \omega / \gamma & \left.J_{a}^{a}+p J_{a}\right] & 0 \\
0 & p \frac{J_{b}}{M} & i \omega / \gamma & 2 \frac{2}{M}\left[K+K_{\mathrm{eff}}^{b}+p J_{b}\right] \\
-p \frac{J_{b}}{M} & 0 & -p \frac{2}{M}\left[K+p J_{b}\right] & i \omega / \gamma
\end{array}\right]\left[\begin{array}{c}
A_{x} \\
A_{y} \\
B_{x} \\
B_{y}
\end{array}\right]=-\left[\begin{array}{c}
0 \\
M h \\
0 \\
M h
\end{array}\right] .
$$

When solutions of the form given in Eqs. (67)-(70) are used to construct the torque equations, one obtains equations of motion for domain-wall oscillations:

$$
\left[\begin{array}{cccc}
i \omega / \gamma & \frac{2}{M}\left[K_{\mathrm{eff}}^{a}+p J_{a}\right] & 0 & -\frac{J_{a}}{M} \\
-\frac{2}{M}\left[K_{D}+p J_{a}\right] & i \omega / \gamma & \frac{J_{a}}{M} & 0 \\
0 & -p \frac{J_{b}}{M} & i \omega / \gamma & p \frac{2}{M}\left[K_{\mathrm{eff}}^{b}+p J_{b}\right] \\
p \frac{J_{b}}{M} & 0 & -p \frac{2}{M}\left[K_{D}+p J_{b}\right] & i \omega / \gamma
\end{array}\right]\left[\begin{array}{c}
A_{x} \\
A_{y} \\
B_{x} \\
B_{y}
\end{array}\right]=-\left[\begin{array}{c}
0 \\
M h \\
0 \\
M h
\end{array}\right]
$$

*Permanent address: Dept. of Physics, Ohio State University, Columbus, OH 43210.

${ }^{1}$ M. Rührig, R. Schäfer, A. Hubert, R. Mosler, J. A. Wolf, S. Demokritov, and P. Grünberg, Phys. Status Solidi A 124, 635 (1991).

${ }^{2}$ L. J. Heyderman, H. Niedoba, H. O. Gupta, and I. B. Puchalska, J. Magn. Magn. Mater. 96, 125 (1991).

${ }^{3}$ J. C. Slonczewski, J. Appl. Phys. 55, 2536 (1984).

${ }^{4}$ H. Braun and O. Brodbeck, Phys. Rev. Lett. 70, 3335 (1993).

${ }^{5}$ P. Grünberg, R. Schreiber, Y. Pang, M. B. Brodsky, and H. Sowers, Phys. Rev. Lett. 57, 2442 (1986).

${ }^{6}$ S. S. P. Parkin, Phys. Rev. Lett. 67, 3598 (1991).

${ }^{7}$ Z. Zhang, L. Zhou, P. E. Wigen, and K. Ounadjela, Phys. Rev.
Lett. 73, 336 (1994).

${ }^{8}$ P. Kabos, C. E. Patton, M. O. Dima, D. B. Church, R. L. Stamps, and R. E. Camley, J. Appl. Phys. 75, 3553 (1994).

${ }^{9}$ B. Heinrich, Z. Celinski, J. F. Cochran, A. S. Arrott, K. Myrtle, and S. T. Purcell, Phys. Rev. B 47, 5077 (1993).

${ }^{10}$ R. L. Stamps, Phys. Rev. B 49, 339 (1994).

${ }^{11}$ A. P. Malozemoff and J. C. Slonczewski, in Applied Solid State Science, edited R. Wolfe (Academic, London, 1979).

${ }^{12}$ J. M. Winter, Phys. Rev. 124, 452 (1961).

${ }^{13}$ S. Middelhoek, J. Appl. Phys. 37, 1276 (1966).

${ }^{14}$ J. C. Slonczewski and S. Middelhoek, Appl. Phys. Lett. 6, 139 (1965). 